\title{
Разработка математического описания электродиализа
}

\author{
Л. В. Равичев ${ }^{1, *}$, С. И. Ильина ${ }^{1, *}$ \\ ${ }^{1}$ Российский химико-технологический университет им. Д. И. Менделеева, \\ Москва, Россия
}

\begin{abstract}
Аннотация
В работе рассмотрен процесс электродиализного разделения как один из методов решения проблем энерго- и ресурсосбережения. Отмечено, что одним из факторов, ограничивающих применение электродиализа, является отсутствие математического описания, что в свою очередь приводит к сложности в планировании проведения процесса. Предложено использовать подход к математическому описанию, основанный на критериальных уравнениях. С этой целью рассмотрены процессы, протекающие при электродиализном разделении, определены их движущие силы. Показано, что если процессы, движущей силой которых является разность концентраций, могут быть описаны с помощью критериев диффузионного подобия, то процессы, протекающие под действием разности потенциалов, могут описываться критериями электрического подобия. Проведен анализ возможности существования таких критериев. Отмечено, что заряд является видом субстанции, и таким образом его перенос может быть описан уравнением переноса субстанции. На основании уравнения переноса заряда можно получить критерии электрического подобия, которые в дальнейшем будут являться определяющими в критериальных уравнениях электро-массообменных процессов, к которым, в частности, относится электродиализ.
\end{abstract}

\section{Ключевые слова}

электродиализ, мембранная технология, уравнение переноса субстанции, критерии подобия, перенос заряда

\section{Введение}

Решение задач энерго- и ресурсосбережения в настоящее время вышло на передний план в мировых масштабах. В этой связи развитие получили мембранные технологии. Движущей силой мембранных методов разделения могут быть разность концентраций, давлений, температур, потенциалов. Также встречаются и сопряженные процессы, в которых различные движущие силы вносят соизмеримый вклад в течение процесса. Примером такого процесса может являться электродиализ (рисунок 1) [1].

Под воздействием электрического поля, когда к электродам приложена разность потенциалов, начинается симметричный процесс. Во время этого процесса катионы движутся к катоду, а анионы в противоположном направлении к аноду. Например, при нумерации отсеков слева направо и расположении анода слева, во время электромиграции к катоду катионы из четных отсеков встречаются на своем 
пути с анионообменной мембраной, которая разделяет нечетный и четный отсеки.

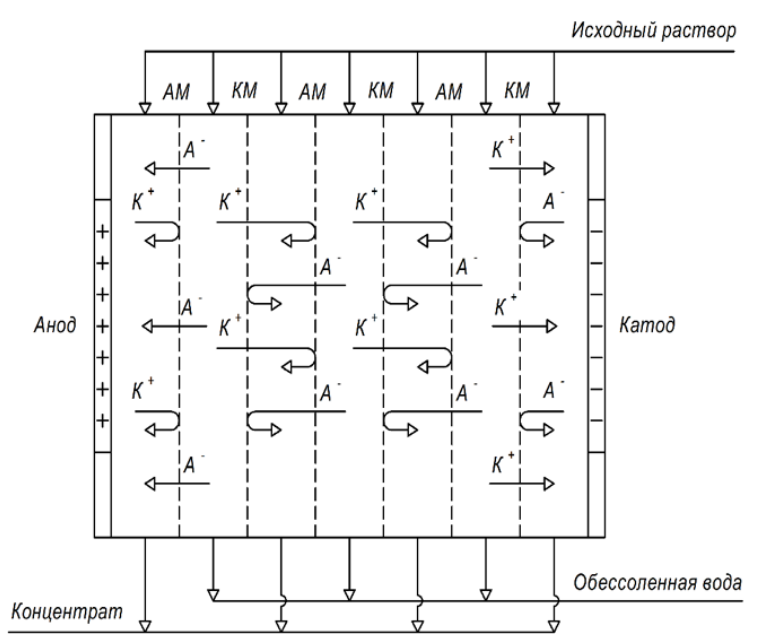

Рисунок 1 - Общая схема процесса электродиализа в многокамерном аппарате.

Эта мембрана останавливает дальнейшую миграцию катионов к катоду. Следовательно, катионы собираются в растворе в нечетном отсеке. С другой стороны, происходит электромиграция анионов из четного отсека в раствор в нечетном отсеке. Эта электромиграция ограничена наличием проницаемой для катионов мембраны, разделяющей четные и нечетные отсеки. Таким образом в четных отсеках происходит уменьшение концентрации ионов (обессоливание раствора), а в нечетных - увеличение ионов (концентрирование раствора).

Первые технологии, включающие электродиализ, появились в конце XIX века, однако, до сих пор ограничены в применении. Одной из причин является отсутствие математического описания процесса, что в свою очередь приводит к невозможности планирования и выбора оптимальных режимов его проведения.

Необходимо отметить, что были предприняты попытки к разработке математического описания [2-6], однако, универсального удобного описания до сих пор нет. Это обусловлено, в основном, тем, что при электродиализном разделении протекают одновременно несколько процессов, а именно: перенос вещества за счет разности потенциалов, перенос вещества за счет разности концентраций, осмоса и электроосмoca.

Математическое описание процесса. Одним из выражений, используемых для расчетов электромембранных процессов, является классическое уравнение Нернста - Планка, описывающее перенос ионов в поле электрохимического потенциала в стационарном режиме:

$$
J=-D \cdot \frac{d c}{d x}-z \cdot F \cdot t_{g} \cdot c \cdot \frac{d u}{d x},
$$

где D - коэффициент диффузии, z — валентность иона, F - число Фарадея, $t_{g}$ - подвижность иона, с - молярная концентрация, u — электрический потенциал.

Первое слагаемое фактически является выражением первого закона Фика и определяет перенос ионов за счет градиента концентраций, в то время как второе слагаемое учитывает перенос ионов за счет разности потенциалов.

Однако в этом уравнении ограниченно представлен конвективный перенос, кроме этого, уравнение не учитывает изменения характеристик переноса во времени.

В связи с этим было принято решение использовать другой подход к построению математического описания.

Как было отмечено ранее, наблюдается два вида переноса: перенос массы и перенос заряда.

Расчет массообменных процессов проводят на основании уравнения конвективной диффузии:

$$
\begin{aligned}
\frac{d c}{d t}+w_{x} \cdot \frac{d c}{d x}+ & w_{y} \cdot \frac{d c}{d y}+w_{z} \cdot \frac{d c}{d z}= \\
& =D \cdot\left(\frac{d^{2} c}{d x^{2}}+\frac{d^{2} c}{d y^{2}}+\frac{d^{2} c}{d z^{2}}\right)= \\
& =D \cdot \nabla^{2} c
\end{aligned}
$$

Часто в технологических расчетах это уравнение является базовым для определения безразмерных величин - критериев 
протекания процессов массопередачи. Однако, как известно, на протекание массообмена влияют и гидродинамические характеристики, в связи с чем критериальные уравнения содержат и критерии гидродинамического подобия, полученные в результате подобного преобразования уравнений Навье-Стокса:

$$
N u_{D}=f\left(R e, P r_{D}, F o_{D}\right)
$$

Вид функции в значительной степени зависит от условий, при которых происходит конкретный процесс. Например, для стационарных процессов исключается критерий $F o_{D}$, содержащий временной параметр. Если в описываемом объеме имеются зоны или точки с разными плотностями, то в набор определяющих критериев вводится критерий Архимеда $A r=g l^{3} \Delta \rho / \rho v^{2}$, также для характеристики геометрии аппаратов и каналов вводятся критерии геометрического подобия $\Gamma 1, \Gamma 2, \ldots$

Используя перечисленные критерии и учитывая стационарность процесса, диффузионный критерий Нуссельта можно представить с другом виде:

$$
N u_{D}=f\left(R e, P r_{D}, A r, \Gamma_{1}, \Gamma_{2}, \ldots\right)
$$

Логично, что так как электродиализ является массообменным процессом, то, соответственно, возможно составить критериальные уравнения, адекватно описывающие протекание процесса в электродиализном аппарате. Однако для этого необходимо сформировать критерии, характеризующие электрическую составляющую процесса. Как показано в работе [7] можно представить зависимость электрического сопротивления в модуле от безразмерной величины $i / i_{\text {пр }}$ (рисунок 2$)$.

Эта зависимость косвенно характеризует перенос ионов электролита на разных стадиях поляризации.

Основываясь на результатах экспериментов, а также на графиках, представленных в литературе [8-10], нами была отмечена следующая зависимость сопротивления в модуле от плотности тока.

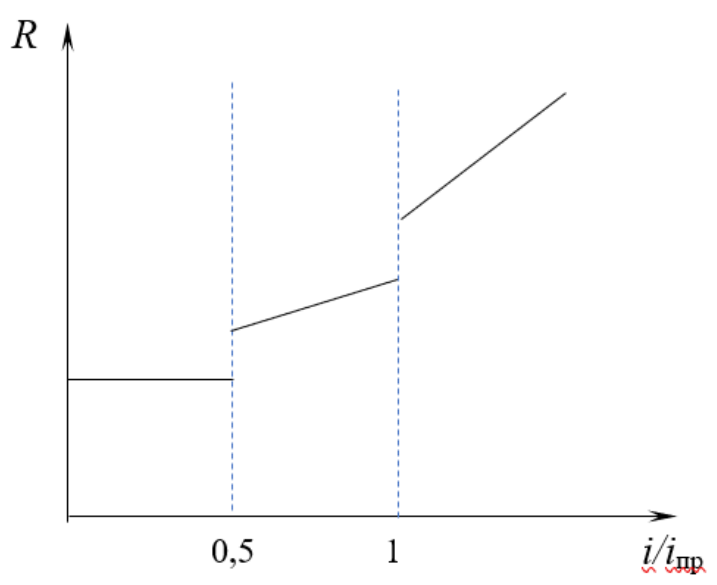

Рисунок 2 - Зависимость электрического сопротивления в модуле от безразмерной величины $i / i_{\text {пр }}$.

$$
\begin{array}{ccl}
\text { При } & \mathrm{i} / \mathrm{i}_{\text {пр }}<0.5 & \mathrm{R}=\text { const, } \\
\text { при } & 0,5<\mathrm{i} / \mathrm{i}_{\text {пр }}<1 & \mathrm{R}=\mathrm{ai}+\mathrm{b} \\
\text { при } & \mathrm{i} / \mathrm{i}_{\text {пр }}>1 & \mathrm{R}=\mathrm{a}^{\prime} \mathrm{i}+\mathrm{b}
\end{array}
$$

Нужно заметить, что скачки сопротивления могут зависеть от конструкции аппарата, геометрии канала, гидродинамики потока, концентрации раствора.

При изменении сопротивления как функции от плотности тока могут происходить изменения следующих лимитирующих стадий процесса.

В первом случае при $\left(i / i_{\text {пр }}>0.5\right)$ протекание процесса определяется скоростью переноса ионов соли через мембрану, это можно утверждать, опираясь на то, что количество ионов, переносимых через мембрану, прямо пропорционально плотности тока. Следовательно, при низких рабочих плотностях тока в растворе существует избыток ионов электролита.

В случае $\mathrm{i} / \mathrm{i}_{\text {пр }}>1$, наоборот, протекание процесса определяется скоростью подвода ионов раствора к поверхности мембраны. При их недостатке ток осуществляется за счет переноса ионов $\mathrm{H}^{+}$и $\mathrm{OH}^{-}$, образующихся при расщеплении воды под действием приложенного напряжения. В этом случае энергия расходуется на 
непроизводительный транспорт, что ведет к резкому увеличению энергозатрат на единицу массы перенесенной соли.

На основании проведенных исследований можно сделать вывод, что при $0.5<\mathrm{i} / \mathrm{i}_{\text {пр }}<1$ процесс переноса ионов контролируется как скоростью переноса ионов соли через мембрану, так и скоростью подвода ионов раствора к поверхности мембраны.

Необходимо также заметить, что во всех случаях, рассмотренных выше, под концентрацией и распределением ионов в растворе подразумевалась концентрация и распределение ионов в толщине диффузионного слоя.

Анализируя эту зависимость, можно провести аналогию с гидромеханическими процессами, где гидравлическое сопротивление жидкой (или газообразной) фазы выступает функцией безразмерного комплекса (критерия Рейнольдса). Причем необходимо отметить, что в гидромеханике также выделяют три области, хотя автомодельная область наблюдается при высоких значениях критерия Re, в то время как в рассматриваемой зависимости автомодельность наблюдается при низких значениях соотношения рабочей и предельной плотностей тока. Таким образом, критерий $\mathrm{i} / \mathrm{i}_{\text {пр }}$ может в электродиализе играть роль своеобразного критерия Рейнольдса.

Как упоминалось ранее, диффузионные и гидродинамические критерии подобия получаются путем преобразования дифференциальных уравнений конвективной диффузии и Навье - Стокса. В свою очередь эти уравнения могут быть получены из основного уравнения переноса субстанции.

$$
\partial \varphi / \partial \tau=-\operatorname{div} \vec{\jmath}+\gamma
$$

где $\varphi$ - потенциал переноса, а именно, отнесенная к единице объема рассматриваемая субстанция; г — время; j — плотность потока субстанции, складывающаяся из переноса на молекулярном (микро-) уровне и конвективном (макро-) уровне; $\gamma$ - удельная объемная плотность притока.

Размерности величин варьируются в зависимости от переносимой субстанции.

Здесь стоит отметить, что уравнение Нернста - Планка также не противоречит уравнению переноса субстанции с учетом, что перенос ионов за счет разности электрических потенциалов будет характеризовать удельную объемную плотность притока $(\gamma)$ при переносе массы.

На основании вышесказанного можно сделать вывод, что, получив уравнение переноса заряда (т. к. заряд тоже является субстанцией) и преобразовав его, можно сформировать критерии электрического подобия. По аналогии с уравнениями переноса массы, тепла и импульса, используя те же условные обозначения, что и в уравнении (1), запишем:

$$
\partial \rho_{\mathrm{v}} / \partial \tau=-\operatorname{div} \overrightarrow{\mathrm{J}_{q}}+\gamma_{q},
$$

где $\rho_{\mathrm{v}}$ - плотность заряда; $\left[\rho_{\mathrm{v}}\right]=\left[\mathrm{Kл} / \mathrm{m}^{3}\right]$, $\left[\gamma_{q}\right]=\left[\mathrm{Kл} / \mathrm{M}^{3} \cdot \mathrm{c}\right]$.

В плотность потока субстанции в уравнении переноса заряда (2) входят две составляющие, представленные в уравнениях (3) и (4):

$$
\overrightarrow{J_{q}}=\overrightarrow{J_{q_{\mathrm{M}}}}+\overrightarrow{J_{q_{\mathrm{K}}}}
$$

В случае протекания процесса на молекулярном уровне, плотность тока заряда описывается уравнением электропроводности:

$$
\overrightarrow{J_{q_{\mathrm{M}}}}=-\sigma \frac{\partial u}{\partial n^{\prime}}
$$

где $\left[\overrightarrow{J_{\mathrm{m}}}\right]=\left[\frac{\text { Кл }}{{\mathrm{c} \cdot \mathrm{M}^{2}}^{2}}\right] ; \sigma-$ удельная электропроводность, $\left[\frac{\mathrm{Cm}}{\mathrm{m}}\right]$.

Конвективную составляющую можно выразить:

$$
\overrightarrow{J_{q_{\mathrm{\kappa}}}}=\vec{\omega} \cdot \rho_{\mathrm{V}}
$$


где $\overrightarrow{J_{q_{\mathrm{K}}}}$ также имеет размерность $\left[\frac{\mathrm{M}}{\mathrm{c}} \cdot \frac{\kappa л}{\mathrm{M}^{3}}\right]=\left[\frac{\kappa л}{\mathrm{c}^{\prime} \mathrm{M}^{2}}\right]$.

Проведя аналогию между тепловыми и электрическими процессами и используя анализ размерностей, появляется возможным преобразовывать уравнение (2) в различные виды с последующим получением критериев электрического подобия, например, электрический критерий Прандтля:

$$
\operatorname{Pr}_{q}=\frac{\mathrm{c}_{m} \cdot \mu}{\sigma}
$$

где $c_{m}$ - удельная ёмкость [Ф/кг], $\mu$ - коэффициент динамической вязкости [Па.с].

Итак, на основании вышеизложенного можно сделать вывод, что для описания электромембранных процессов также можно использовать критериальный подход.

Помимо критериев диффузионного и электрического подобия, в уравнение также должен входить критерий Рейнольдса, так как концентрационная поляризация мембран непосредственно зависит от режима течения жидкости, а именно, предельная плотность тока зависит от толщины диффузионного слоя ( $\delta)$ :

$$
i_{\text {пр }}=\frac{z F c}{\bar{t}_{g}-t_{g}}\left(\frac{D}{\delta}+\frac{4 \bar{D} c}{X d}\right),
$$

где $\mathrm{X}$ - число фиксированных ионов в мембране; $t_{g}$ - подвижность иона в растворе; $\bar{t}_{g}$ - подвижность иона в мембране; $\mathrm{D}$ - коэффициент диффузии в растворе; $\bar{D}$ — коэффициент диффузии в мембране; d - толщина мембраны.

Кроме этого необходимо учитывать конфигурацию канала, размеры турбулизаторов и т.п.

Тогда для стационарного сопряженного электро-массообменного процесса можно предположить следующую функцию:

$$
N u_{D}=f\left(R e, P r_{D}, P r_{q}, i / i_{\text {пр }}, \Gamma_{1}, \Gamma_{2} \ldots\right) .
$$

\section{Заключение}

В заключение стоит обобщить вышесказанное:

Так как электрический ток относится к явлениям переноса, то для описания процессов, проходящих под действием разности потенциалов, можно использовать уравнение переноса субстанции.

Следует отметить, что, так как уравнение основано на базовых законах и определениях, помимо получения критериев электрического подобия, может стать универсальным методом описания процессов, связанных с переносом заряда.

\section{СПИСОК ЛИТЕРАТУРЫ}

[1]. Быков В. И., Ильина С.И., Логинов В. Я., Равичев Л. В., Свитцов А. А. Электродиализ: история и перспективы развития. Вестник Технологического университета. 2021. Т.24. №7. С. 5-10

[2]. Кононов А. В. Моделирование сверхпредельного массопереноса в процессе электродиализа промышленных стоков. В книге: XXVIII Менделеевская конференция молодых ученых. Сборник тезисов. 2018. C. 65.

[3]. Заболоцкий В. И., Лебедев К. А., Василенко П. А., Васильева В. И., Шапошник В. А., Жильцова А. В. Математическое моделирование влияния электроконвекции на вольтамперные кривые и числа переноса в запредельных режимах электродиализа. Сорбционные и хроматографические процессы. 2012. Т. 12. № 3. C. $332-337$.

[4]. Шапошник В. А., Васильева В. И., Григорчук О. В. Теоретическое и экспериментальное моделирование электродиализа. В сборнике: Проблемы химии и химической технологии. 1998. С. 58-64. 
[5]. Гнусин Н. П. Моделирование электромассопереноса в электродиализной ячейке. Теор. основы хим. технол. 2004. T. 38. № 3. С. 316-320.

[6]. Коваленко А. В., Никоненко В. В., Уртенов М. А. Х., Лойко В. И. Физический смысл некоторых критериев подобия процесса переноса в канале обессоливания электродиализного аппарата с учетом электроконвекции. Политематический сетевой электронный научный журнал Кубанского государственного аграрного университета. 2015. № 105. С. 846-865.

[7]. Ильина С. И. Процессы обессоливания и концентрирования в однопоточных электродиализаторах: Диссертация на соискание ученой степени кандидата технических наук. М., 2001. 113 с.

[8]. Бесман В. Л. Гидродинамические и поляризационные характеристики ЭДА лабиринтного типа: Диссертация на соискание ученой степени кандидата технических наук. Алма-Ата. 1969.

[9]. Заболоцкий В. И., Никоненко В. В. Перенос ионов в мембранах. М., «Наука», 1996. 392 c.

[10]. Гнусин Н. П. Электродиффузионный перенос в электродиализной ячейке, работающей в режиме обессоливания и концентрирования солевых растворов. Режим допредельного состояния. Кинетическая задача. Электрохимия. 1999. Т. 35, № 6. С. $747-752$.

Равичев Леонид Владимирович - д-р техн. наук, зав. кафедрой процессов и аппаратов химической технологии РХТУ им. Д. И. Менделеева (Российская Федерация, 125047, Москва, Миусская площадь, д.9).

Ильина Светлана Игоревна - канд. техн. наук, доцент кафедры процессов и аппаратов химической технологии РХТУ им. Д. И. Менделеева (Российская Федерация, 125047, Москва, Миусская площадь, д.9). 


\title{
Development of a mathematical description of electrodialysis
}

\author{
L. V. Ravichev ${ }^{1, *}$, S. I. Ilyina ${ }^{1, *}$ \\ ${ }^{1}$ Mendeleev University of Chemical Technology of Russia, Moscow, Russia
}

\begin{abstract}
The paper considers the process of electrodialysis separation as one of the methods for solving problems of energy and resource conservation. It is noted that one of the factors limiting the use of electrodialysis is the lack of a mathematical description, which in turn leads to difficulties in planning the process. It is proposed to use an approach to mathematical description based on criteria equations. For this purpose, the processes occurring during electrodialysis separation are considered, their driving forces are determined. It is shown that if the processes, the driving force of which is the concentration difference, can be described using the criteria of diffusion similarity, then the processes occurring under the action of the potential difference can be described by the criteria of electrical similarity. The analysis of the possibility of the existence of such criteria is carried out. It is noted that the charge is a type of substance, and thus its transfer can be described by the mass transfer equation. Based on the series transfer equation, it is possible to obtain criteria of electrical similarity, which in the future will be decisive in the criteria equations of electro-mass transfer processes, which include electrodialysis.
\end{abstract}

\section{Keywords}

Electrodialysis, membrane technology, substance transfer equation, similarity criteria, charge transfer

\section{REFERENCES}

[1]. Bykov V.I., Il'ina S.I., Loginov V.Ja., Ravichev L.V., Svitcov A.A. Electrodialysis: History and development prospects. Vestnik Tehnologicheskogo universiteta. 2021. T.24. №7, pp. 5-10. (In Russ.).

[2]. Kononov A.V. Modeling of over-thelimit mass transfer in the process of electrodialysis of industrial effluents. XXVIII Mendeleev Conference of Young Scientists. Collection of abstracts. 2018, p. 65. (In Russ.).

[3]. Zabolockij V.I., Lebedev K.A., Vasilenko P.A., Vasil'eva V.I., Shaposhnik V.A., Zhil'cova A.V. Mathematical modeling of the effect of electroconvection on currentvoltage curves and transfer numbers in exorbitant electrodialysis modes. // Sorption and chromatography processes. 2012. T. 12. № 3, pp. 332-337. (In Russ.).

[4]. Shaposhnik V.A., Vasil'eva V.I., Grigorchuk O.V. Theoretical and experimental modeling of electrodialysis. // Problems of chemistry and chemical technology. 1998, pp. 58-64. (In Russ.).

[5]. Gnusin N. P. Modeling of mass electrotransfer in an electrodialysis cell. // Theoretical Foundations of Chemical Engineering. 2004. V. 38, № 3, pp. 296-300.

[6]. Kovalenko A.V., Nikonenko V.V., Urtenov M.A.H., Lojko V.I. The physical meaning of some criteria for the similarity of the transfer process in the desalination channel of the electrodialysis apparatus, taking into 
account electroconvection. // Polythematic online scientific journal of Kuban state agrarian university .2015. № 105, pp. 846865. (In Russ.).

[7]. Il'ina S.I. Desalination and concentration processes in single-flow electrodialysts.: Thesis for the degree in Eng. Sc. M.: 2001. 113 p. (In Russ.).

[8]. Besman V.L. Hydrodynamic and polarization characteristics of labyrinth-type
ED.: Thesis for the degree in Eng. Sc. Alma-Ata. 1969. (In Russ.).

[9]. Zabolockij V.I., Nikonenko V.V. Ion transfer in membranes. - M.: «Nauka». 1996. - 392 p. (In Russ.).

[10]. Gnusin N.P. Eelectrodiffusion transfer in electrodialysis cells operating in the mode of desalination and concentration of salt solutions: the sublimiting mode and the kinetic problem. // Russian journal of electrochemistry. 1999. V. 35, № 6, pp. $669-674$.

Ravichev L. V. - Dr. Sc. (Eng.), Head of the Department of Processes and Devices of Chemical Technology of D.I. Mendeleev Russian State Technical University (Miusskaya square, 9, Moscow, 125047, Russian Federation).

Ilyina S. I. - Cand. Sc. (Eng.), Assoc. Professor, Department of Processes and Devices of Chemical Technology of D.I. Mendeleev Russian State Technical University (Miusskaya square, 9, Moscow, 125047, Russian Federation).

\section{Просьба ссылаться на эту статью следующим образом:}

Разработка математического описания электродиализа / Л. В. Равичев, С. И. Ильина // Промышленные процессы и технологии. 2021. № 2. С. 62 - 69 .

DOI: $10.37816 / 2713-0789-2021-1-2-62-69$

\section{Please cite this article as:}

Ravichev L. V., Ilyina S. I. Development of a mathematical description of electrodialysis. Industrial processes ang Technologies, 2021, no. 2, pp. $62-69$

DOI: $10.37816 / 2713-0789-2021-1-2-62-69$ 